\title{
Coefficient Estimates and Bloch's Constant in Some Classes of Harmonic Mappings
}

\author{
S. Kanas ${ }^{1}$ • D. Klimek-Smęt ${ }^{2}$
}

Received: 15 September 2013 / Revised: 14 November 2013 / Published online: 15 July 2015

C) The Author(s) 2015. This article is published with open access at Springerlink.com

\begin{abstract}
Following Clunie and Sheil-Small, the class of normalized univalent harmonic mappings in the unit disk is denoted by $\mathcal{S}_{\mathcal{H}}$. The aim of the paper is to study the properties of a subclass of $\mathcal{S}_{\mathcal{H}}$, such that the analytic part is a convex function. We establish estimates of some functionals and bounds of the Bloch's constant for co-analytic part.
\end{abstract}

Keywords Univalent harmonic mappings - Convex functions · Bloch's constant . Normal family

Mathematics Subject Classification $\quad 30 \mathrm{C} 75 \cdot 30 \mathrm{C} 45 \cdot 30 \mathrm{D} 45$

\section{Introduction}

A complex-valued harmonic function $f$ that is harmonic in a simply connected domain $\Omega \subset \mathbb{C}$ has the canonical representation

Communicated by V. Ravichandran.

S. Kanas

skanas@ur.edu.pl

D. Klimek-Smęt

dominika@hektor.umcs.lublin.pl

1 Faculty of Mathematics and Natural Sciences, University of Rzeszow, ul. S. Pigonia 1, 35-959 Rzeszów, Poland

2 Department of Mathematics, Maria Curie-Sklodowska University in Lublin, Pl. Marii Curie-Skłodowskiej 5, 20-031 Lublin, Poland 


$$
f=h+\bar{g},
$$

where $h$ and $g$ are analytic in $\Omega$ with $g\left(z_{0}\right)=0$ for some prescribed point $z_{0} \in$ $\Omega$. According to a theorem of Lewy [17], $f$ is locally univalent, if and only if its Jacobian $J_{f}(z)=\left|f_{z}(z)\right|^{2}-\left|f_{\bar{z}}(z)\right|^{2}=\left|h^{\prime}(z)\right|^{2}-\left|g^{\prime}(z)\right|^{2}$ does not vanish, and is sense-preserving if the Jacobian is positive. Then $h^{\prime}(z) \neq 0$ and the analytic function $\omega=g^{\prime} / h^{\prime}$, called the second complex dilatation of $f$, has the property $|\omega|<1$ in $\Omega$. Throughout this paper, we will assume that $f$ is locally univalent and sense-preserving, and we call $f$ a harmonic mapping. Also, we assume $\Omega=\mathbb{D} \subset \mathbb{C}$, and $z_{0}=0$, where $\mathbb{D}$ is the open unit disk on the complex plane. The class of all sense-preserving univalent harmonic mappings of $\mathbb{D}$ with $h(0)=g(0)=h^{\prime}(0)-1=0$ is denoted by $\mathcal{S}_{\mathcal{H}}$, and its subclass for $g^{\prime}(0)=0$ by $\mathcal{S}_{\mathcal{H}}^{0}$ (cf. [8]). Fundamental informations about harmonic mappings in the plane can be found in [11]. Note that each $f$ satisfying (1.1) in $\mathbb{D}$ is uniquely determined by coefficients of the following power series expansions

$$
h(z)=\sum_{n=0}^{\infty} a_{n} z^{n}, \quad g(z)=\sum_{n=1}^{\infty} b_{n} z^{n} \quad(z \in \mathbb{D}),
$$

with $a_{n} \in \mathbb{C}, n=0,1,2, \ldots$, and $b_{n} \in \mathbb{C}, n=1,2,3, \ldots$ When $f \in \mathcal{S}_{\mathcal{H}}$, then $a_{0}=0, a_{1}=1$.

In [14], the authors studied the properties of a subclass $\overline{\mathcal{S}}_{\mathcal{H}}^{\alpha}$ of $\mathcal{S}_{\mathcal{H}}$, consisting of all univalent anti-analytic perturbations of the identity in the unit disk with $\left|b_{1}\right|=\alpha$, and in [15], the authors studied the class $\widehat{\mathcal{S}}^{\alpha}$ of all $f \in \mathcal{S}_{\mathcal{H}}$, such that $\left|b_{1}\right|=\alpha \in(0,1)$ and $h \in \mathcal{C} \mathcal{V}$, where $\mathcal{C V}$ denotes the well-known family of normalized, univalent functions which are convex.

The classical Schwarz-Pick estimate for an analytic function $\omega$ which is bounded by one on the unit disk of the complex plane is the inequality

$$
\left|\omega^{\prime}(z)\right| \leq \frac{1-|\omega(z)|^{2}}{1-|z|^{2}} \quad(|z|<1) .
$$

Ruscheweyh [21] has obtained the best-possible estimates of higher order derivatives of bounded analytic functions on the disk. Similar estimates were derived by other methods and for different classes of analytic functions in one and several variables by Anderson and Rovnyak [1]

$$
\left(1-|z|^{2}\right)^{n-1}\left|\frac{\omega^{(n)}(z)}{n !}\right| \leq \frac{1-|\omega(z)|^{2}}{1-|z|^{2}} \quad(n=1,2, \ldots) .
$$

The case $z=0$ in (1.4) asserts that if

$$
\omega(z)=c_{0}+c_{1} z+c_{2} z^{2}+\cdots,
$$

then

$$
\left|c_{n}\right| \leq 1-\left|c_{0}\right|^{2},
$$


for every $n \geq 1$. This result is classical and due to Wiener; see $[2,16]$.

\section{Bounds of the Fekete-Szegö and Other Functionals}

Theorem 2.1 Let $f \in \widehat{S}^{\alpha}, f=h+\bar{g}$ with the power series (1.2). Then

$$
\left|b_{n}\right| \leq \alpha+\frac{\left(1-\alpha^{2}\right)(n-1)}{2} \quad(n=2,3, \ldots) .
$$

Proof Making use of a relation $g^{\prime}=\omega h^{\prime}$ and the power series expansions (1.2) and (1.5), we obtain

$$
n b_{n}=\sum_{p=0}^{n-1}(p+1) a_{p+1} c_{n-p-1} \quad(n=2,3, \ldots) .
$$

Since $h \in \mathcal{C} \mathcal{V},\left|a_{k}\right| \leq 1(k=1,2, \ldots)$. Applying this for $(2.2)$, we have

$$
\left|b_{n}\right| \leq \frac{1}{n} \sum_{p=0}^{n-1}(p+1)\left|c_{n-p-1}\right|
$$

The fact $g^{\prime}=\omega h^{\prime}$, for the case $z=0$, implies that $c_{0}=b_{1}$, so that by (1.6), we obtain $\left|c_{n-p-1}\right| \leq 1-\left|b_{1}\right|^{2}=1-\alpha^{2}$. Therefore,

$$
\begin{aligned}
\left|b_{n}\right| & \leq \alpha+\frac{1}{n} \sum_{p=0}^{n-2}(p+1)\left(1-\alpha^{2}\right) \\
& =\alpha+\frac{\left(1-\alpha^{2}\right)(n-1)}{2} .
\end{aligned}
$$

Specially, we get

$$
\left|b_{2}\right| \leq \alpha+\frac{1-\alpha^{2}}{2}, \quad\left|b_{3}\right| \leq 1+\alpha-\alpha^{2} .
$$

For the case $n=2$, the inequality is sharp, with the equality realized by the function

$$
f(z)=\frac{z}{1-z}+\overline{\frac{z}{1-z}-\frac{1-\alpha}{1+\alpha} \log \frac{1+\alpha z}{1-z}} .
$$

We note that for $\alpha$ close to 1 , the above bounds are better than that obtained in [15]. 
In conclusion, we obtain

Corollary 2.2 Let $f \in \widehat{S}^{\alpha}, f=h+\bar{g}$ with the power series (1.2). Then

$$
\left|b_{n}\right| \leq \min \left\{\alpha+\frac{\left(1-\alpha^{2}\right)(n-1)}{2}, \frac{\alpha+\sqrt{\left(n-\alpha^{2}\right)(n-1)}}{n}\right\} \quad(n=2,3, \ldots) .
$$

Theorem 2.3 Let $f \in \widehat{S}^{\alpha}, f=h+\bar{g}$ with the power series (1.2). Then for $\mu \in \mathbb{R}$

$$
\left|b_{3}-\mu b_{2}^{2}\right| \leq \frac{1-\alpha^{2}}{3}\left\{1+\frac{3}{4}|\mu|\left(1-\alpha^{2}\right)+\left|2-3 \mu b_{1}\right|\right\}+\alpha \max \left\{\frac{1}{3},\left|1-\mu b_{1}\right|\right\}
$$

and

$$
\left|b_{n+1}-b_{n}\right| \leq 2 \alpha+\left(1-\alpha^{2}\right) \frac{2 n-1}{2}
$$

Proof From the relation (2.2), we have

$$
2 b_{2}=c_{1}+2 a_{2} c_{0}, \quad 3 b_{3}=c_{2}+2 a_{2} c_{1}+3 a_{3} c_{0} .
$$

Then

$$
\begin{aligned}
\left|b_{3}-\mu b_{2}^{2}\right| & =\left|\frac{1}{3} c_{2}+\frac{2}{3} a_{2} c_{1}+a_{3} c_{0}-\mu\left(\frac{1}{2} c_{1}+a_{2} c_{0}\right)^{2}\right| \\
& =\left|\left(\frac{1}{3} c_{2}-\frac{\mu}{4} c_{1}^{2}\right)+a_{2} c_{1}\left(\frac{2}{3}-\mu c_{0}\right)+c_{0}\left(a_{3}-\mu c_{0} a_{2}^{2}\right)\right| \\
& \leq \frac{1}{3}\left|c_{2}-\frac{3}{4} \mu c_{1}^{2}\right|+\left|a_{2}\right|\left|c_{1}\right|\left|\frac{2}{3}-\mu c_{0}\right|+\alpha\left|a_{3}-\mu c_{0} a_{2}^{2}\right| .
\end{aligned}
$$

Apply now the estimate that holds for the coefficients of convex functions: $\left|a_{n}\right| \leq$ $1(n=2,3, \ldots),\left|a_{3}-v a_{2}^{2}\right| \leq \max \{1 / 3,|1-v|\}(v \in \mathbb{R})$, and the relation (1.6). We obtain then

$$
\begin{aligned}
\left|b_{3}-\mu b_{2}^{2}\right| \leq & \frac{1}{3}\left[\left|c_{2}\right|+\frac{3}{4}\left|\mu c_{1}^{2}\right|\right]+\left|a_{2}\right|\left|c_{1}\right|\left|\frac{2}{3}-\mu c_{0}\right|+\alpha\left|a_{3}-\mu b_{1} a_{2}^{2}\right| \\
\leq & \frac{1-\alpha^{2}}{3}\left[1+\frac{3}{4}|\mu|\left(1-\alpha^{2}\right)\right]+\left(1-\alpha^{2}\right)\left|\frac{2}{3}-\mu b_{1}\right| \\
& +\alpha \max \left\{1 / 3,\left|1-\mu b_{1}\right|\right\} .
\end{aligned}
$$


Next, by (2.2), we have

$$
\begin{aligned}
\left|b_{n+1}-b_{n}\right| & =\left|\frac{1}{n+1} \sum_{p=1}^{n+1} p a_{p} c_{n+1-p}-\frac{1}{n} \sum_{p=1}^{n} p a_{p} c_{n-p}\right| \\
& \leq\left|a_{n+1} c_{0}\right|+\left|a_{n} c_{0}\right|+\frac{1}{n+1} \sum_{p=1}^{n} p\left|a_{p} c_{n+1-p}\right|+\frac{1}{n} \sum_{p=1}^{n-1} p\left|a_{p} c_{n-p}\right| \\
& \leq 2 \alpha+\frac{1-\alpha^{2}}{n+1} \sum_{p=1}^{n} p+\frac{1-\alpha^{2}}{n} \sum_{p=1}^{n-1} p \\
& =2 \alpha+\left(1-\alpha^{2}\right) \frac{2 n-1}{2} .
\end{aligned}
$$

The proof is now complete; however, the results are not sharp, for example, the function that realizes the accuracy of $\left|b_{2}\right|$ in the previous theorem gives $\left|b_{2}-b_{1}\right|=\left(1-\alpha^{2}\right) / 2 \leq$ $2 \alpha+\left(1-\alpha^{2}\right) / 2$, for any $\alpha \in(0,1)$. The right-hand side is obtained from (2.6) for the case when $n=1$.

Theorem 2.4 For $f \in \widehat{S}^{\alpha}, f=h+\bar{g}$ and $|z|=r<1$, it holds

$$
\begin{gathered}
\left|\frac{r}{1+r}-\frac{1-\alpha}{1+\alpha} \log \frac{1+r}{1-\alpha r}\right| \leq|g(z)| \leq \frac{r}{1-r}+\frac{1-\alpha}{1+\alpha} \log \frac{1-r}{1+\alpha r} \\
\frac{2 r}{1+r}-\frac{r\left(1-\alpha^{2}\right)}{|r-\alpha|(1-\alpha r)} \leq\left|\frac{z g^{\prime \prime}(z)}{g^{\prime}(z)}\right| \leq \frac{2 r}{1-r}+\frac{r\left(1-\alpha^{2}\right)}{|r-\alpha|(1-\alpha r)} \\
\operatorname{Re}\left(1+\frac{z g^{\prime \prime}(z)}{g^{\prime}(z)}\right)>\frac{r\left(\alpha^{2}-1\right)}{|\alpha-r|(1-\alpha r)}+\frac{1-r}{1+r}
\end{gathered}
$$

Proof Applying the relation $g^{\prime}=\omega h^{\prime}$, we estimate $\left|g^{\prime}(z)\right|$ as follows [15]:

$$
\frac{|\alpha-r|}{(1-\alpha r)(1+r)^{2}} \leq\left|g^{\prime}(z)\right| \leq \frac{\alpha+r}{(1+\alpha r)(1-r)^{2}} \quad(|z|=r<1)
$$

Then integrating along a radial line $\zeta=t e^{i \theta}$, the right-hand side of (2.7) is obtained immediately [15].

In order to prove the left-hand side of (2.7), we note first that $g$ is univalent. Let $\Gamma=g(\{z:|z|=r\})$ and let $\xi_{1} \in \Gamma$ be the nearest point to the origin. By a rotation we may assume that $\xi_{1}>0$. Let $\gamma$ be the line segment $0 \leq \xi \leq \xi_{1}$ and suppose that $z_{1}=g^{-1}\left(\xi_{1}\right)$ and $L=g^{-1}(\gamma)$. With $\zeta$ as the variable of integration on $L$, we have that $\mathrm{d} \xi=g^{\prime}(\zeta) \mathrm{d} \zeta>0$ on $L$. Hence 


$$
\begin{aligned}
\xi_{1} & =\int_{0}^{\xi_{1}} \mathrm{~d} \xi=\int_{0}^{z_{1}} g^{\prime}(\zeta) \mathrm{d} \zeta=\int_{0}^{z_{1}}\left|g^{\prime}(\zeta)\right||\mathrm{d} \zeta| \geq \int_{0}^{r}\left|g^{\prime}\left(t e^{i \theta}\right)\right| \mathrm{d} t \\
& \geq \int_{0}^{r} \frac{|\alpha-r|}{(1-\alpha r)(1+r)^{2}} \mathrm{~d} r=\left|\frac{r}{1+r}-\frac{1-\alpha}{1+\alpha} \log \frac{1+r}{1-\alpha r}\right| .
\end{aligned}
$$

From the relation $g^{\prime}=\omega h^{\prime}$, we obtain

$$
\frac{z g^{\prime \prime}(z)}{g^{\prime}(z)}=\frac{z \omega^{\prime}(z)}{\omega(z)}+\frac{z h^{\prime \prime}(z)}{h^{\prime}(z)} .
$$

Since $h$ is convex, so is univalent, then it holds [13, p. 118]

$$
\frac{2 r}{1+r} \leq\left|\frac{z h^{\prime \prime}(z)}{h^{\prime}(z)}\right| \leq \frac{2 r}{1-r} \quad(|z|=r) .
$$

Moreover, $\omega$ satisfies [12, p. 320]

$$
\left|\frac{\omega(z)-\omega(0)}{1-\overline{\omega(0)} \omega(z)}\right| \leq|z| \quad(|z|=r),
$$

from which it follows

$$
\left|\omega(z)-\frac{\omega(0)\left(1-r^{2}\right)}{1-|\omega(0)|^{2} r^{2}}\right| \leq \frac{r\left(1-|\omega(0)|^{2}\right)}{1-|\omega(0)|^{2} r^{2}} .
$$

We note that $|\omega(0)|=\left|c_{0}\right|=\left|b_{1}\right|=\alpha$, so that by (2.13) we have

$$
\frac{|r-\alpha|}{1-\alpha r} \leq|\omega(z)| \leq \frac{r+\alpha}{1+\alpha r}
$$

Taking into account (2.10), (2.11), and (2.14) and the Schwarz-Pick inequality (1.3), we obtain for $|z|=r<1$,

$$
\begin{aligned}
\left|\frac{z g^{\prime \prime}(z)}{g^{\prime}(z)}\right| & \leq\left|\frac{z \omega^{\prime}(z)}{\omega(z)}\right|+\left|\frac{z h^{\prime \prime}(z)}{h^{\prime}(z)}\right| \\
& \leq \frac{r\left(1-|\omega(z)|^{2}\right)}{|\omega(z)|\left(1-r^{2}\right)}+\frac{2 r}{1-r} \\
& \leq \frac{r\left(1-r^{2}\right)\left(1-\alpha^{2}\right)}{\left(1-r^{2}\right)|r-\alpha|(1-\alpha r)}+\frac{2 r}{1-r} \\
& =\frac{r\left(1-\alpha^{2}\right)}{|r-\alpha|(1-\alpha r)}+\frac{2 r}{1-r} .
\end{aligned}
$$


Similarly, we have

$$
\begin{aligned}
\left|\frac{z g^{\prime \prime}(z)}{g^{\prime}(z)}\right| & \geq\left|\frac{z h^{\prime \prime}(z)}{h^{\prime}(z)}\right|-\left|\frac{z \omega^{\prime}(z)}{\omega(z)}\right| \\
& \geq \frac{2 r}{1+r}-\frac{r\left(1-|\omega(z)|^{2}\right)}{|\omega(z)|\left(1-r^{2}\right)} \\
& \geq \frac{2 r}{1+r}-\frac{r\left(1-r^{2}\right)\left(1-\alpha^{2}\right)}{\left(1-r^{2}\right)|r-\alpha|(1-\alpha r)} \\
& =\frac{2 r}{1+r}-\frac{r\left(1-\alpha^{2}\right)}{|r-\alpha|(1-\alpha r)} .
\end{aligned}
$$

Moreover

$$
1+\frac{z g^{\prime \prime}(z)}{g^{\prime}(z)}=\frac{z \omega^{\prime}(z)}{\omega(z)}+1+\frac{z h^{\prime \prime}(z)}{h^{\prime}(z)}
$$

and $h$ is convex, therefore

$$
\begin{aligned}
\operatorname{Re}\left(1+\frac{z g^{\prime \prime}(z)}{g^{\prime}(z)}\right) & =\operatorname{Re} \frac{z \omega^{\prime}(z)}{\omega(z)}+\operatorname{Re}\left(1+\frac{z h^{\prime \prime}(z)}{h^{\prime}(z)}\right) \\
& >\operatorname{Re} \frac{z \omega^{\prime}(z)}{\omega(z)}+\frac{1-r}{1+r}
\end{aligned}
$$

By the above, (1.3) and (2.14), we have

$$
\operatorname{Re}\left(1+\frac{z g^{\prime \prime}(z)}{g^{\prime}(z)}\right)>\frac{r\left(\alpha^{2}-1\right)}{|\alpha-r|(1-\alpha r)}+\frac{1-r}{1+r},
$$

as asserted.

\section{Estimates of the Bloch's Constant}

A harmonic function $f$ is called the Bloch function if

$$
\mathfrak{B}_{f}=\sup _{z, w \in \mathbb{D}, z \neq w} \frac{|f(z)-f(w)|}{\varrho(z, w)}<\infty,
$$

where

$$
\varrho(z, w)=\frac{1}{2} \log \left(\frac{1+\left|\frac{z-w}{1-\bar{z} w}\right|}{1-\left|\frac{z-w}{1-\bar{z} w}\right|}\right)=\operatorname{artanh}\left|\frac{z-w}{1-\bar{z} w}\right|
$$

denotes the hyperbolic distance in $\mathbb{D}$, and $\mathfrak{B}_{f}$ is called the Bloch's constant of $f$. The harmonic Bloch's constant was studied by Colonna [9]. Colonna established that the 
Bloch's constant $\mathfrak{B}_{f}$ of a harmonic mapping $f=h+\bar{g}$ can be expressed in terms of moduli of the derivatives of $h$ and $g$

$$
\begin{aligned}
\mathfrak{B}_{f} & =\sup _{z \in \mathbb{D}}\left(1-|z|^{2}\right)\left(\left|h^{\prime}(z)\right|+\left|g^{\prime}(z)\right|\right) \\
& =\sup _{z \in \mathbb{D}}\left(1-|z|^{2}\right)\left|h^{\prime}(z)\right|(1+|\omega(z)|),
\end{aligned}
$$

which agrees with the well-known notion of the Bloch's constant for analytic functions. Moreover, the function $f$ is Bloch if and only if both $h$ and $g$ are, and

$$
\max \left(\mathfrak{B}_{h}, \mathfrak{B}_{g}\right) \leq \mathfrak{B}_{f} \leq \mathfrak{B}_{h}+\mathfrak{B}_{g}
$$

Colonna also obtained the best-possible estimate of the Bloch's constant for the family of harmonic mappings of $\mathbb{D}$ into itself. Recently, the Bloch's constant was studied by many authors, see, for example $[3,4,19]$. Very interesting results in this direction were obtained in $[5-7,18,20,22]$. Our aim is to determine the bounds for the Bloch's constant in the classes $\bar{S}^{\alpha}$ and $\widehat{S}^{\alpha}$.

Theorem 3.1 Let $f=h+\bar{g}$ with $h(z)=z /(1-B z),-1<B<1$, and let $|B|=A, 0 \leq A<1$. Then the Bloch's constant $\mathfrak{B}_{f}$ is bounded by

$$
\mathfrak{B}_{f} \leq(1+\alpha) \frac{\left(1+r_{0}\right)^{3}\left(1-r_{0}\right)^{2}}{\left(1-A r_{0}\right)^{2}\left(1+\alpha r_{0}\right)}
$$

where $r_{0}$ is given by

$$
r_{0}=\frac{\alpha(1+3 A)-3-A+\sqrt{(1+\alpha)(1+A)(9-7 A+\alpha(-7+9 A))}}{4 \alpha+2 A(\alpha-1)} .
$$

Proof Applying the distortion theorem

$$
\left|h^{\prime}(z)\right| \leq \frac{1}{(1-A r)^{2}} \quad(|z|=r)
$$

and (3.2), we find

$$
\mathfrak{B}_{f}=\sup _{z \in \mathbb{D}}\left(1-|z|^{2}\right)\left|h^{\prime}(z)\right|(1+|\omega(z)|) \leq(1+\alpha) \sup _{0 \leq r<1} \frac{(1+r)\left(1-r^{2}\right)}{(1-A r)^{2}(1+\alpha r)} .
$$

Setting

$$
q(r)=\frac{(1+r)\left(1-r^{2}\right)}{(1-A r)^{2}(1+\alpha r)}
$$

we observe that $q^{\prime}(r)=0$, if and only if

$$
(1+r)\left[(2 \alpha+\alpha A-A) r^{2}+(3+A-\alpha-3 \alpha A) r+\alpha-1-2 A\right]=0 .
$$


The last equation has solution in the interval $(0,1)$ at the point $r_{0}$ given by $(3.4)$, and the function $q$ attains its maximum at $r_{0}$.

Setting $B=0$ in the above theorem, we obtain the estimate of $\mathfrak{B}_{f}$ in the class $\bar{S}^{\alpha}$, below.

Corollary 3.2 For $f \in \bar{S}^{\alpha}, f=h+\bar{g}$, the Bloch's constant $\mathfrak{B}_{f}$ is bounded by

$$
\mathfrak{B}_{f} \leq(1+\alpha) \frac{\left(1+r_{0}\right)^{3}\left(1-r_{0}\right)^{2}}{\left(1+\alpha r_{0}\right)}
$$

where $r_{0}$ is given by

$$
r_{0}=\frac{\alpha-3+\sqrt{9+2 \alpha-7 \alpha^{2}}}{4 \alpha} .
$$

Remark By the fact that the Bloch's constant is finite, we already have that the family of harmonic mappings with $h(z) \equiv z$, and $\left|b_{1}\right|=\alpha$, is a normal family. A function $f$ is normal, if the constant $\sigma_{f}$ is finite, where

$$
\sigma_{f}=\sup _{z \in \mathbb{D}} \frac{\left(1-|z|^{2}\right)\left|f^{\prime}(z)\right|}{1+|f(z)|},
$$

see [10]. Indeed, since the quantity $|f(z)|$ in $\overline{\mathcal{S}}^{\alpha}$ is bounded [15]

$$
|f(z)| \geq \begin{cases}\left(1-\frac{1}{\alpha}\right) r-\left(1-\frac{1}{\alpha^{2}}\right) \log (1+\alpha r) & \text { for } \alpha \neq 0, \\ r-\frac{r^{2}}{2} & \text { for } \alpha=0,\end{cases}
$$

therefore, by (3.5) and (3.7), we obtain

$$
\sigma_{f} \leq \begin{cases}(1+\alpha) \frac{(1+r)\left(1-r^{2}\right)}{1+\alpha r} \frac{1}{1+\left(1-\frac{1}{\alpha}\right) r-\left(1-\frac{1}{\alpha^{2}}\right) \log (1+\alpha r)} & \text { for } \alpha \neq 0 \\ (1+\alpha) \frac{(1+r)\left(1-r^{2}\right)}{1+\alpha r} \frac{1}{1+r-\frac{r^{2}}{2}} & \text { for } \alpha=0\end{cases}
$$

where $r=r_{0}$ is given by (3.6), and we see that in both cases $\sigma_{f}$ is finite.

Remark The univalent Bloch functions can be described in terms of geometry of their images; they are precisely those functions whose images do not contain disks of arbitrarily large radius [10]. Therefore, we suppose that the functions from the class $\widehat{S}^{\alpha}$ may not be the Bloch functions. Indeed, reasoning similarly as in the Theorem 3.1 we note that in the class $\widehat{S}^{\alpha}$ we have $h(z)=z /(1-z)$, then $\left|h^{\prime}(z)\right| \leq 1 /(1-r)^{2}$. Thus

$$
\mathfrak{B}_{f}=(1+\alpha) \sup _{0 \leq r<1} \frac{(1+r)^{2}}{(1-r)(1+\alpha r)}
$$


and the function $p(r)=(1+r)^{2} /[(1-r)(1+\alpha r)]$ increases in the whole interval $(0,1)$, with infinity as the supremum.

Acknowledgments This work was partially supported by the Centre for Innovation and Transfer of Natural Sciences and Engineering Knowledge.

Open Access This article is distributed under the terms of the Creative Commons Attribution 4.0 International License (http://creativecommons.org/licenses/by/4.0/), which permits unrestricted use, distribution, and reproduction in any medium, provided you give appropriate credit to the original author(s) and the source, provide a link to the Creative Commons license, and indicate if changes were made.

\section{References}

1. Anderson, J.M., Rovnyak, J.: On generalized Schwarz-Pick estimates. Mathematika 53, 161-168 (2006)

2. Bénéteau, C., Dahlner, A., Khavinson, D.: Remarks on the Bohr phenomenon. Comput. Methods Funct. Theory 4(1), 1-19 (2004)

3. Chen, H., Gauthier, P.M., Hengartner, W.: Bloch constants theorem for planar harmonic mappings. Proc. Am. Math. Soc. 128, 3231-3240 (2000)

4. Chen, Sh, Ponusamy, S., Wang, X.: Bloch constant and Landau's theorem for planar p-harmonic mappings. J. Math. Anal. Appl. 373, 102-110 (2011)

5. Chen, Sh, Ponnusamy, S., Wang, X.: Coefficient estimates and Landau-Bloch's constant for planar harmonic mappings. Bull. Malays. Math. Sci. Soc. 34, 255-265 (2011)

6. Chen, Sh, Ponnusamy, S., Wang, X.: Landau's theorem and Marden constant for harmonic v-Bloch mappings. Bull. Aust. Math. Soc. 84, 19-32 (2011)

7. Chen, Sh, Ponnusamy, S., Vuorinen, M., Wang, X.: Lipschitz spaces and bounded mean oscillation of planar harmonic mappings. Bull. Aust. Math. Soc. 88, 143-157 (2013)

8. Clunie, J.G., Sheil-Small, T.: Harmonic univalent functions. Ann. Acad. Sci. Fenn. Ser. A. I. Math. 9, 3-25 (1984)

9. Colonna, F.: The Bloch constant of bounded harmonic mappings. Indiana Univ. Math. J. 38, 829-840 (1989)

10. Colonna, F.: Bloch and normal functions and their relation. Rend. Circ. Mat.Palermo, Serie II, Tomo XXXVIII, 161-180 (1989)

11. Duren, P.L.: Harmonic Mappings in the Plane, Cambridge Tracts in Mathematics 156. Cambridge University Press, Cambridge (2004)

12. Goluzin, G.M.: Geometric Theory of Functions of a Complex Variable, Izdatelstwo Nauka, Moscow (1969) (English transl. Math. Soc. Am. 1969)

13. Goodman, A.W.: Univalent Functions. Mariner Publishing, Tampa (1983)

14. Klimek, D., Michalski, A.: Univalent anti-analytic perturbations of the identity in the unit disc. Sci. Bull. Chełm 1, 67-76 (2006)

15. Klimek D., Michalski A.: Univalent anti-analytic perturbations of convex analytic mappings in the unit disc Ann. Univ. Mariae Curie-Skodowska Sect. A, Vol. LXI, Sectio A, 39-49 (2007)

16. Landau, E., Gaier, D.: Darstellung und Begründung einiger neuerer Ergebnisse der Funktionentheorie. Springer, Berlin (1986)

17. Levy, H.: On the non-vanishing of the Jacobian in certain one-to-one mappings. Bull. Am. Math. Soc. 42, 689-692 (1936)

18. Li, P., Ponnusamy, S., Wang, X.: Some properties of planar p-harmonic and log-p-harmonic mappings. Bull. Malays. Math. Sci. Soc 36(3), 595-609 (2013)

19. Liu, M.: Estimates on Bloch constants for planar harmonic mappings. Sci. China Ser. A 52, 87-93 (2009)

20. Rashidah, O., Suzeini, A.H.: Multivalent harmonic functions defined by Dziok-Srivastava operator. Bull. Malays. Math. Sci. Soc. 35(3), 601-610 (2012)

21. Ruscheweyh, St: Two remarks on bounded analytic functions. Serdica 11(2), 200-202 (1985)

22. Subramanian, K.G., Stephen, B.A., Lee, S.K.: Subclasses of multivalent harmonic mappings defined by convolution. Bull. Malays. Math. Sci. Soc. 35(3), 717-726 (2012) 